\title{
DONNE CHE SI SACRIFICANO NELLA GRECIA ANTICA: ALCESTI
}

\author{
WOMEN THAT SACRIFICED THEMSELVES IN ANCIENT GREECE: ALCESTIS
}

Daniele Cerrato

Universidad de Sevilla

\section{Riassunto:}

Nella Grecia del V secolo a.C. era molto frequente che le donne venissero sacrificate in onore della patria. Polissena nell'Ecuba di Euripide, Ifigenia nell'Ifigenia in Aulide e Macaria negli Eraclidi sono tre esempi di vergini costrette al sacrificio. Un caso a parte è quello di Alcesti: il suo sacrificio non consiste solo nella distruzione della donna, ma della relazione che ha con suo marito: avviene lo sconvolgimento di un ordine coniugale.

\section{Parole chiave:}

Alcesti, donna, sacrificio.

\section{Abstract:}

In Greece during the 5th century B.C., it was very common for women to be sacrificed in honour of the country. Polyxena in Hecuba by Euripides, Iphigenia in Aulis and Macaria in the Children of Heracles, are three examples of virgin women forced to sacrifice. Alcestis' sacrifice is a whole different story. Her sacrifice it wasn't only about destroying her, but also about the relationship with her husband: the marital order collapsed.

\section{KEY WORD:}

Alcestis, woman, sacrifice. 
Sacrificare (dal lat. composto di sacrum e facere) significa letteralmente rendere sacro. In Grecia, sacri erano i doni o le vittime che si offrivano agli dei, per cercare di stabilire un rapporto con loro. Chi si sacrifica è chi si separa da qualcosa che gli appartiene e mette in atto un processo di annientamento della propria persona.

Le donne greche del V secolo a.C vivono in una società che ne fa sovente un capro espiatorio. La loro è una rinuncia e una privazione quotidiana. Si tratta di donne educate all'inferiorità e che hanno fatto della sottomissione un'abitudine e un'attitudine. La tragedia greca diventa proiezione e specchio di questo mondo. Le protagoniste spesso sono presentate come donne al limite, donne isteriche e depresse, donne esasperate $\mathrm{e}$ che non hanno scelta, per le quali prendere decisioni estreme diventa quasi un obbligo. Spesso l'unica via di fuga, ma al tempo stesso di riscatto, sembra essere per loro quella del sacrificio. Il sacrificio si trasforma in un dovere e una responsabilità con rispetto ai parenti, oppure alla comunità che glielo richiede. Il sacrificio può dare gloria, luce e onore ad una vita e solo la morte gloriosa può risollevare totalmente la donna. È il caso delle vergini che vengono sacrificate, in nome della patria e dei valori maschili. I loro sacrifici ricordano molto da vicino quelli degli animali immolati sulle are sacre. Anche la terminologia e i riferimenti spesso sono i medesimi. La loro vita e il loro sangue puro, offerti in sacrificio agli dei, diventano un esempio da innalzare alle generazioni successive, da ricordare e incidere nel tempo (Cfr. Loraux, 1985).

Il sacrificio delle vergini può arrivare a sostituire l'onore del matrimonio. L'associazione sacrificio-matrimonio è infatti una costante, sia per quanto riguarda Polissena nell'Ecuba di Euripide, sia nel caso di Ifigenia nell'Ifigenia in Aulide, sia per Macaria negli Eraclidi. Così Polissena, che fantasticava per lei un fastoso matrimonio da regina, si ritrova a morire come una schiava, trascinata di fronte all'esercito greco che aspetta di salpare. Però è proprio nella morte che la giovane vergine crede di trovare la propria consacrazione e compimento, la libertà che oramai le sembrava preclusa. Sull'altare Polissena si lacera da sola la veste, mostra i suoi seni, offre il petto e il collo al sacrificio. Quando la spada penetra nella sua carne, e il sangue comincia a sgorgare copiosamente, si preoccupa di cadere composta, per conservare anche nella morte la sua dignità e il suo onore. La donna greca deve sempre mantenere un rigore e un onore, anche negli ultimi respiri della sua vita, come la società del suo tempo le impone. I sacrifici delle vergini diventano nozze rovesciate. Cosí accade nell'Ifigenia in Aulide di Euripide. Agamennoneinganna la figlia, facendole credere che andrá in sposa ad Achille. Presso l'altare non si celebrerá il suo matrimonio ma si consumerá il suo sacrificio. Altro caso esemplare è quello di Macaria che, negli Eraclidi, si offre come vittima per salvare i fratelli. Macaria sceglie di morire perché pensa che una vergine sola, senza nessuno che la protegga, non possa affrontare la vita. La morte, sembra essere, ancora una volta, l'unica strada. Macaria muore, e l'unica speranza che le resta è quella di ritagliarsi uno spazio nei ricordi dei fratelli. Per Polissena, Ifigenia e Macaria il sacrificio diventa riscatto personale, un riconoscimento, la possibilità di ottenere la considerazione o, almeno, il rispetto dell'universo maschile. Sono peró le circostanze a costringerle a scegliere questa strada. Tutte e tre sono figlie di un mondo creato da uomini e costruito interamente su valori maschili, dove il sacrificio rientra all'interno delle mansioni del femminile. Un caso particolare è invece quello di Alcesti.

Apollo $^{1}$ ha concesso ad Admeto, re di Fere, di evitare la morte a patto che qualcuno si sacrifichi al suo posto. Tutti sembrano rifiutare e sarà Alcesti, la sua sposa, a sacrificarsi e a morire. Admeto avrà salva la vita. Nel finale della tragedia Eracle, dopo essere sceso all'Ade, riconsegnerà Alcesti ad Admeto, ma lei rimarrà coperta da un velo $\mathrm{e}$ in silenzio. Questa in breve la trama della tragedia, ma, dietro l'apparente semplicità dell'intreccio, sono molti i risvolti e le pieghe che meritano di essere analizzate con maggiore attenzione.

Innanzitutto Alcesti non è una vergine, anzi ha dei figli, una posizione e dei privilegi importanti. I critici hanno però spesso idealizzato Alcesti in una visione romantica di eroina che muore per amore ${ }^{2}$. Alcesti non può però essere identificata solo come una sposa modello ${ }^{3}$ che, per preservare il marito, affronta senza timore la morte. Come Polissena, Ifigenia e Macaria, Alcesti sceglie il sacrificio perché le è richiesto socialmente, lo sceglie perchè la norma lo prevede. Nel suo caso, il sacrificio riuscirà a

1 Il rapporto di filia di Admeto con gli dei ha origini remote e ha inizio, come ci raccontano Apollodoro e altri mitografi, per un debito contratto da Apollo con Zeus. Apollo è condannato a servire Admeto per un determinato periodo di tempo. L'ospitalità offerta da Admeto (Euripide si sofferma molto su questo tema nel primo dialogo Admeto- Eracle) gli fara guadagnare la riconoscenza di Apollo, che 10 e, tra Admeto e Eracle poi, vi sia qualcosa di più di una forte amicizia, quasi un sentimento di amore. e, tra Admeto e Eracle poi, vi sia qualcosa di piu di una forte amicizia, quasi un sentimento di amore. Cosi Tibullo: Pavit et Admeti tauros formosus Apollo,/ nec cithara intonsae profueruntve comae, nec potuit curas sanare salubribus herbis:/ quicquid erat medicae vicerat artis amor./ ("Perfino i bell'Apollo pascolò i tori di Admeto,/ non potendo giovargli la cetra e i riccioli intonsi;/ né egli poté sanare le sue pene con erbe salutari,/ avendo l'amore vinto ogni ritrovato dell'arte medica. (Albii Tibulli aliorumque carminum libri tres, libro II, elegia 3, vv. 11-14 e segg.; ctr. anche III (Lygdami elegiarum liber), elegia 4, vv. 67-72.) Cosi invece Plutarco : "Si narra che Eracle, esperto in medicina,
intervenne a salvare Alcesti quando la donna era in condizioni disperate, per far cosa gradita ad intervenne a salvare Alcesti quando la donna era in condizioni disperate, per far cosa gradita ad pure Apollo divenne l'amante di Admeto e si pose al suo servizio, per un anno intero (Plutarco, 'Er?tikós, 761e.).

2 La morte della donna al posto del marito, soprattutto il giorno delle nozze è un tema antico che si trova sviluppato in molte fiabe popolari e leggende. Un importante contributo a proposito è quello $d$ A. Lesky (Alkestis, der Mythus und das Drama, "Sitzungsberichte der Akademie der Wissenshaften in Wien" 203.2, 1925, pp. 1-86. G. Paduano in Euripide, Alcesti, La Nuova Italia, Firenze 1969, p. VII, sottolinea come soprattutto in area germanica si trovino casi in cui è l'uomo a sacrificarsi per la

3 L'immagine di Alcesti come sposa modello che si sacrifica per amore, e che attraverso le parole di Fedro viene anteposta per meriti anche ad Orfeo, si trova anche nel simposio di Platone. "Soltanto gli amanti giungono a morire per l'altro. Non solamente uomini, anche donne. Figlia di Pelia, Alcesti ne ha dato ai greci un esempio luminoso". Platone, Sympósion, 179b-d. 
sbilanciare il rapporto con il marito a favore suo e, a stravolgere, o almeno, mettere in discussione il suo ruolo di sposa ${ }^{4}$.

La vicenda di Alcesti va dunque interpretata sempre tenendo ben presente la netta dicotomia del rapporto uomo/donna che si evidenzia nell'Atene del V secolo. Vita fuori di casa per l'uomo e vita chiusa tra le mura domestiche per la donna. Spazi e opportunità, contrapposti a limitazioni e divieti. Il teatro, a volte, permette di accorciare questa distanza, dar voce al dissenso, permette alle donne di entrare in un terreno che nella vita quotidiana gli è precluso. Se si analizza con attenzione la tragedia, perché di tragedia si tratta e non di tragicommedia, come alcuni studiosi hanno sostenuto (Cfr. Del Grande, 1962), sembra quindi emergere un preciso tentativo da parte di Euripide di modificare gli equilibri di questo rapporto, smontandone e rovesciandone le caratteristiche fondamentali, di deviare, attraverso la tragedia, il modo di pensare comune. Se il principio del sacrificio è la distruzione, come avevamo detto all'inizio, in questo caso non si tratta solo della distruzione della persona di Alcesti, ma della relazione che ha con suo marito. Come sostiene U. Galimberti, i sacrificio sconvolge un ordine, pone fine ai legami per stabilire un vuoto (Galimberti, 2000 : 21), quel vuoto che risuonerà nel silenzio di Alcesti quando tornerà dalla morte. In ogni caso quel vuoto è già presente in tutto il testo che appare costruito su due blocchi differenti. In tutta la tragedia c'è un solo dialogo tra marito e moglie e, anche in questa occasione, i protagonisti sembrano percorrere strade parallele che mai si intersecano. Ognuno mette in scena una sorta di monologo in presenza dell'altro. L'amore e il lessico d'amore sono quasi del tutto assenti e, il rapporto Admeto-Alcesti a tratti appare estremamente freddo, quasi un rapporto contrattualistico. Dopo la morte della moglie, Admeto non pronuncierà più il suo nome, salvo nel finale della tragedia (Cfr. Neri, 1992). Quando poi, Eracle li riunirà, sarà Alcesti a restare in silenzio, come a sottolineare che la distanza creatasi tra di loro è ormai incolmabile. In apertura di tragedia una serva ci racconta come la protagonista si lavi, indossi il vestito più bello e scelga i gioielli più preziosi. Sembra prepararsi alla morte con calma e tranquillità, come seguendo un rituale già predisposto. Tutto fino a quando, ritornata nella sua stanza, si trova di fronte il letto nuziale. Il letto sembra essere lo strumento, l'immagine che le permette di acquisire la consapevolezza e la percezione del suo sacrificio.

"Y después se fue a su alcoba y a su lecho

donde llora y dice:

Oh lecho donde perdí mi virginidad

con este hombre por quien muero,

4 A proposito del sacrificio di Alcesti in rapporto agli altri sacrifici presenti nelle opere euripidee si veda ad esempio Valgiglio, E., Il tema della morte in Euripide, Biblioteca della Rivista di Stud Classici, Saggi vari no4, Torino, 1966. "Confrontato con il sacrificio di Ifigenia, il sacrificio di Alcesti è meno grandioso e meno ricco però più sofferto e più sentito, più costoso, meno spettacolare ma più intimo, meno ideale ma più vicino alla realtà, meno sublime ma più comune." jadiós! No te odio: solo a mí me matas: por no

traicionarte a ti y a mi marido,

muero. Te serás de otra mujer

no más fiel que yo, más feliz quizas"

La morte di Alcesti si produce in questo momento: morte simbolica che sopraggiunge con la consapevolezza che il marito ama di più la propria vita rispetto a lei. Se è vero, da una parte, che questa circostanza concede più valore al sacrificio di Alcesti (Brillante, 2005), dall'altra, fa ricadere su di lei tutto il peso della mancanza di reciprocità nel loro legame d'amore, poiché lei ama di più il marito della propria vita. Davanti al suo letto si risveglia dal suo sogno di moglie felice, la discesa all'Ade è solo un passaggio successivo, una morte corporale. Questa è già la sua catabasi e la sua catarsi. In questo episodio riesce a guardarsi "da fuori", anticipando anche quello che sarà il futuro del marito senza di lei. Alcesti ora può morire e uscire di scena perché si è resa conto del suo stato, della situazione che sta vivendo. Alcesti, con il sacrificio, non solo mette in discussione il proprio ruolo ma, di conseguenza, anche il ruolo di Admeto. Vuole fare sì che la sua non sia solo una "belle mort" ma, qualcosa che possa trasformarsi in un beneficio concreto, almeno per i figli. $\mathrm{Si}$ assiste quindi a una sorta di trattativa, a una negoziazione, uno scambio.

"Admeto, ves cómo estan mis asuntos,

quiero decirte antes de morir, lo que deseo.

Yo, que te he procurado en cambio de mi vida

ver este dia, voy a morir por tí cuando podria no hacerlo

y cuando podria encontrar el marido tesalio que quisiera

y tener una feliz morada señorial.

No he querido vivir privada de tí

con mis niños huerfanos, ni guardo avara,

mi juventud, con los dones que yo disfrutaba".

Per Alcesti, dopo una vita di sacrificio al servizio del marito, è giunta l'ora di presentargli il conto. Il capovolgimento dei ruoli può dirsi completo. Alcesti non è più donna che accompagna, ma donna che guida, e da cui ora il marito dipende ed è indissolubilmente legato. Il discorso di Alcesti diventa preciso e ordinato, con argomentazioni puntuali e attente considerazioni. La donna che piangeva davanti al suo letto e si faceva travolgere dai ricordi e dai rimpianti, ha lasciato il posto a una donna decisa e risoluta, che non sembra più farsi influenzare e condizionare dal marito. I riferimenti alla vita passata insieme scompaiono completamente.

Ad Admeto, che le chiede che cosa potrà fare una volta restato solo, risponde (El tiempo te calmarà: el muerto es nada). E poco più avanti, quanto Admeto le chiede di portarlo con lei nell' Ade (Ya basto yo, la que muero por ti). La distanza sembra acuirsi sempre più, poco prima attraverso una sorta di cerimoniale, Alcesti aveva preso per mano i figli, e li aveva affidati ad Admeto chiedendogli di far loro da madre: “Hijos,

5 Tutte le traduzioni dei testi di Euripide in spagnolo sono personali. 
estais oyendo lo que vuestro padre dice que no se casará con otra mujer, por vosotros y por no deshonrarme... Entonces recibe estos niños de mi mano... Sé tu ahora, en mi lugar, madre de estos niños". L'appellativo "donna" viene spesso usato nella tragedia greca come termine dispregiativo ${ }^{6}$ nei confronti di un uomo e Alcesti, chiedendo al marito di farsi madre, lo trascina fuori dalle tipiche funzioni che spettano ad un uomo e a un re del suo tempo. Anche il coro, si domanda se per Admeto non sarebbe meglio suicidarsi, attraverso un impiccagione, modo di morire tipico dei personaggi tragici femminili che si tolgono la vita ${ }^{7}$. Alcesti invece si fa uomo: è lei che scende agli Inferi perché Admeto possa restare a vedere la luce. ? lei a prendere l'iniziativa, ad agire. Se Orfeo era sceso all'Ade per recuperare Euridice, ora è Alcesti che riscatta Admeto:

“Tú, mujer única y querida,

cambiaste el alma de tu marido

por la tuya cuando

ya estaba en el Hades".

Successivamente, sará lo stesso Admeto a definire Alcesti come madre e padre insieme, evidenziando un mutamento nei ruoli classici imposti dalla società greca all'uomo e alla donna ("esta mujer extraña, a la cual yo, madre y padre, podria con razon creer mía"). Alcesti è dunque un'eroina che demolisce gli stereotipi, trasforma Admeto da campione del genos a madre e custode dell' oikos. Il sacrificio permette ad Alcesti di imporsi, di far sentire la sua voce, di assumere il comando, costringere il marito a giurare, a sottomettersi. Alcesti fa quello che una donna greca del V secolo mai si sarebbe potuta permettere, imporre la propria legge al marito, collocandola al di sopra della norma sociale, come aveva fatto Antigone. Alcesti viene ad incarnare tutti i valori di cui Admeto sembra essere privo, da donna di casa si trasforma in donna di azione, in esempio di virtù. Admeto, dopo aver chiesto alla moglie di sacrificarsi per lui, resta ancorato ai se, ("si yo tuviera la lengua y el canto de Orfeo $\left.{ }^{8 \prime \prime}\right)$, e alla sua passività. Admeto ha rifiutato la propria morte e si specchia in quella della moglie, cercando di appropriarsi anche del suo dolore. Admeto forse potrebbe ancora cambiare la situazione, salvare la moglie e morire ma, nelle sue parole c'è solo riconoscenza e ringraziamento, quindi accettazione e rassegnazione.

Per Admeto, come prevede il codice della società greca, la sua compagna, non è solo una donna di carne ed ossa, ma soprattutto una merce di scambio. La morte di una moglie, come si è detto, non è mai un dramma paragonabile a quella dí un uomo o di un familiare dello stesso sangue. La morte di una donna pesa meno, puô essere assorbita e superata con maggiore disinvoltura. Euripide illustra una sorta di gerarchia delle morti. La vita umana non viene considerata come un valore assoluto, ma come un valore del tuto relativo. Nei due incontri con Admeto anche Eracle, quando si infoma su chi sia morto, nomina prima i figli, il padre e la madre di Admeto, Alcesti, la moglie, è sempre in fondo a questo elenco di possibili lutti.

Admeto una volta morta Alcesti, abbandona presto il ruolo del vedovo per riassumere prontamente quello di re, che, con grande lucidità, dà disposizioni precise per allestire il funerale. Il ruolo, la sua funzione all'interno della

6 Così lo utilizza Eschilo nelle Eumenidi per riferirsi a Egisto per sottolineare la sua incapacità decisionale.

7 Il suicidio è un atto che può incontrare il rispetto degli uomini ma resta pur sempre una morte da donne, un gesto che denota mancanza di coraggio. Anche nella scelta del modo di suicidarsi vi è una distinzione di sessi. Aiace e Emone scelgono il pugnale le donne invece, salvo rare eccezion come nel caso di Deianira e Euridice, optano per l'impiccagione

8 Secondo la leggenda, Orfeo aveva partecipato con Admeto ed Eracle alla impresa degli Argonauti. città, la sua immagine agli occhi dei suoi cittadini sono le preoccupazioni di Admeto. Alcesti ha rubato la gloria che spettava all'uomo, al re, al condottiero. Il vero timore di Admeto è quello di essere accusato dai nemici di essere un codardo, di essersi fatto battere da una donna, di non essere un vero uomo. Riesce a riversare questa colpa sul capo di Alcesti, colpevole di averlo fatto sfigurare, di averlo messo in cattiva luce di fronte agli amici. Cosi come arà lui stesso ad ammettere:

“Esta es la fama que entre los malévolos tendré.

¿Para qué, amigos, me glorio de vivir,

con mala fama y mala fortuna?"

Per Admeto, la propria immagine, l'apparenza sono più importanti dell'amore e il suo lutto ha a che vedere con la perdita dei suoi riferimenti domestici. Alcesti le manca come moglie, per il suo ruolo di madre, ma non come donna. Anche nell'incontro finale con Eracle, quando avviene "la restituzione" della moglie, Admeto mostra ancora una volta tutta la sua ambiguità e le sue debolezze. Sembra più preoccupato di ringraziare l'amico che di riabbracciare la moglie. Solo successivamente, Admeto chiede ad Eracle perché Alcesti non parli e alla risposta di Eracle, che appare molto confusa ed enigmatica, resta tranquillo, non fa altre domande, non appare preoccupato. La cosa importante sembra il fatto di essere rientrato in possesso della moglie, come si ritrova un oggetto, smarrito nei sotterranei dell'Ade, che non si pensava di poter riottenere.

Alcesti, invece, non ha scelto di voler tornare, di riunirsi al marito. Il suo ritorno altro non è che un favore tra uomini, un rispettare un patto di onore tra vecchi amici. Non bisogna dimenticare che lo status della donna in Grecia rimane pur sempre quello di un oggetto, Alcesti rappresenta il dono che due potenti si scambiano, cioè diventa il mezzo attraverso il quale i rapporti di potere e gerarchia si rinforzano fra uomini (Beltrametti, 2008). Alcesti ritorna quasi in una dimensione a sè stante, staccata dal resto dei comuni mortali, come se nulla potesse più infrangere lo stato che ha raggiunto. Il senso del sacrificio di Alcesti, si ritrova allora nell'etimologia della parola "sacro" come "separata" rispetto al mondo in cui viveva prima. Accettando di morire e tornando dalla morte, non soltanto rimane in una condizione di isolamento rispetto al resto degli umani, ma, rimane soprattutto estranea a sé stessa, separata da quella che era prima e che adesso non potrà piú tornare ad essere. Questo sdoppiamento del personaggio viene già annunciato lungo tutta l'opera; già Admeto nel primo incontro con Eracle aveva parlato della moglie in questi termini:

"Sobre ella es posible dar doble version...

Está y no está y me causa dolor"

D'altronde, è significativo che lungo tutto il testo si parli di Alcesti come "straniera" e come "donna estranea" . Per fare un parallelo con Medea, possiamo dire che in questi

9 Sul termine estranea è costruito tutto il dialogo tra Eracle ed Admeto.(vv.533) Heracles: Forastera o alguien parienta tuya? Admeto: Forastera mas por otra parte forzosamente emparentada a la casa. La parola serve a tenere in piedi l'equivoco e a non far capire ad Eracle che la donna che dovrà morire Alcesti. Il termine ritornerà nel dialogo tra Eracle e il Servo e servirà a sottolineare la superiorita morale di Alcesti nei confronti degli altri personaggi e la sua condizione di separata. 
personaggi femminili si racchiude la dialettica fra l'identità greca e l'Altro, che è il corpo estraneo che stravolge l'ordine, che rompe i meccanismi dell'identificazione. Alcesti, nella sua dimensione separata, simbolizza proprio questo Altro (Beltrametti, 2008: 47). Una straniera che nessuno sembra capire e allora ha scelto la lingua del silenzio. Se si prova ad analizzare la situazione con gli occhi di Alcesti, il suo silenzio si riempie di contenuti. D'altronde, cosa potrebbe dire Alcesti ad Admeto, all'uomo che ha permesso che morisse per lui, all'uomo che l'ha vista sacrificarsi e non ha fatto nulla per impedirlo? Il suo silenzio sottolinea il suo status di morte simbolica, in contrapposizione alla sua presenza fisica, il suo silenzio é l'assenza da un discorso che soltanto può dirsi in maschile. Dice Maria Zambrano (2001) che Antigone non avrebbe potuto togliersi la vita perché mai l'aveva posseduta realmente, lo stesso succede ad Alcesti che perde la vita per causa di un uomo e, la recupera per causa di un altro. Vá e viene dalla morte come una moneta di scambio. Se Antigone appartiene ad una "stirpe di murati", como sostiene Lola Luna (1996: 12), non lo è di meno Alcesti, circondata dal muro invisibile, ma non meno consistente, del silenzio.

\section{RIFERIMENTI BIBLIOGRAFICI}

Albani, U., “L'Alcesti di Euripide” en Maia 13, Firenze, 1961.

Beltrametti, A., “Ifigenia e le altre. Archetipi greci del sacrificio, oblazione eroica e crimine politico nella cultura ateniese del V secolo" in Storia delle donne, IV, pp. 47-69 Firenze University Press, 2008.

Brillante, C., L'Alcesti di Euripide. Materiali e discussioni per l'analisi dei testi classici, 2005.

Citti, V., Tragedia e lotta di classe in Grecia, Liguori Editore, Napoli, 1996.

Del Grande, C. Tragoidía. Essenza e genesi della tragedia, Milano-Napoli, Ricardo Ricciardi, 1962.

Di Benedetto, V., Euripide: teatro e società, Einaudi, Torino, 1971.

Eurípides, Alceste, Colleción Griegos y Latinos, trad. de Pablo A. Cavallero, Losada, 2007.

Galimberti, U., Sulle orme del sacro, Feltrinelli, Milano, 2000.

Garzya, A., Pensiero e tecnica drammatica in Euripide, Libreria Scientifica Editrice, Napoli, 1962.

Mamolar Sánchez, I. "El sacrificio de Alcestis" in Veleia: Revista de preistoria, istoria antigua, arquelogia y filologia clásica, № 7, pags 243-250, 1990.

Kott, J., "Alcesti velata", in Divorare gli dei. Un'interpretazione della tragedia greca, Bruno Mondadori, Milano 2005.

Lesky, A. "Alkestis, der Mythus und das Drama", Sitzungsberichte der Akademie der Wissenshaften in Wien, 1925.

Loraux, N., Façon tragiques de tuer une femme, Textes du XX Siècle, Hachette, 1985.

Luna, L., Leyendo como una mujer la imagen de la mujer, Anthropos, Barcelona, 1996.
Muller de Inda, C., Consciencia de la responsabilidad: Alcestis y Antigona in Synthesis, (3):19-32, 1996. Neri, A., Per una lettura antropologica dell'Alcesti in Lexis: Poética, retórica e comunicaciones nella tradizione, (9-10): 93-114, 1992

Paduano, G., Euripide, Alcesti, La Nuova Italia, Firenze,1969.

Pattoni, M. P., Alcesti. Variazioni sul mito, Marsilio, Venezia, 2006.

Tagliapietra, A., Il velo di Alcesti. La filosofia e il teatro della morte, Feltrinelli, Milano,1997.

Yourcenar, M., "Esame di Alcesti", in Tutto il teatro, trad. di Luca Coppola e Giancarlo Prati, Bompiani, Milano, (da Théâtre I et Théâtre II, Gallimard, Parigi, 1971), 1997.

Valgiglio, E., Il tema della morte in Euripide, Biblioteca della Rivista di Studi Classici, Saggi vari n4, Torino 1966.

Zambrano, M., La tomba di Antigone, Milano, La Tartaruga, 2001. 